\title{
Original
}

\section{Resultados del estudio metabólico en 54 pacientes con urolitiasis de alto riesgo de recurrencia}

\author{
Enrique Ossandón Salas, Oscar Storme Cabrera, Rodrigo Ledesma, Fernando Marchant González, \\ Cristian Palma Ceppi, Pedro Recabal Guiraldes
}

Servicio de Urología. Unidad de Litiasis. Hospital Clínico de la Universidad de Chile. José Joaquín Aguirre. Chile

\section{Resumen}

Introducción: La urolitiasis es considerada actualmente una enfermedad metabólica con tendencia a la recurrencia. El objetivo de este trabajo es evaluar la prevalencia de alteraciones metabólicas en pacientes de alto riesgo y su impacto según sexo y edad.

Materiales y métodos: Es un estudio descriptivo de 54 pacientes (37 hombres y 17 mujeres), portadores de patología litiásica con alto riesgo de recurrencia. El estudio metabólico consistió en: calcemia, uricemia, fosfemia, PTH sérica, calciuria/24 h, uricosuria/24 h, fosfaturia/24h, oxalaturia/24 h, citraturia/24 h y creatininuria/24 h. Los valores obtenidos fueron ajustados de acuerdo a la creatininuria y peso. Para el análisis estadístico se utilizó t-student ( STATA 7.0). Se consideró significativo $\mathrm{p}<0,05$.

Resultados: En el 64,8\% (35/54) se observó alguna alteración metabólica; el 27,7 \% (15/54) presentó 2 o más alteraciones metabólicas. Las alteraciones más frecuentes fueron la Hipercalciuria (15/54) 27,7\%, Hipocitraturia (15/54) 27,7\%., Hiperuricemia (8/54) 14,8\%. Hiperoxalaturia (8/54) 14,8\%., Hiperuricosuria (7/54) 12,9\%. No se observó diferencias significativas de edad o sexo entre los grupos con y sin alteración metabólica.

Conclusiones: La mayoría de los pacientes con patología litiásica recurrente o de alto riesgo presentan una o más alteraciones metabólicas, predominando la hipercalciuria y la hipocitraturia. En este estudio no hubo diferencias entre ambos sexos en la mayoría de las alteraciones metabólicas, ni tampoco en su distribución etaria. Estos resultados demuestran la necesidad de realizar estudios metabólicos en pacientes de alto riesgo, dado que existen herramientas terapéuticas que permiten un manejo médico de las alteraciones metabólicas y de esta forma reducir la recurrencia de litiasis.

Palabras clave: Urolitiasis. Enfermedad metabólica. Estudio metabólico.

\section{Metabolic study results of 54 patients with high risk of recurrent urolitiasis}

\begin{abstract}
Introduction: Urolithiasis is a metabolic disorder with a tendency to relapse. The aim of this study was to assess the prevalence of metabolic abnormalities in patients at high risk and the impact of sex and age.

Materials and methods: Descriptive study of 54 patients ( 37 men and 17 women),with lithiasic pathology at high risk of recurrence. The metabolic study included the measurement of calcemia, uricemia, fosfemia, parathormone, calciuria/24 h, uricosuria/24 h, fosfaturia/24 h, oxalaturia/24 h, citraturia/24 h and creatinine/24 h. The values obtained were corrected according to weight and creatinine. The test used for statistical analysis was t-student (STATA 7.0). It was considered significant $\mathrm{p}<0.05$.

Results: In $64,8 \%(35 / 54)$ of the cases a metabolic abnormality was observed and in $27,7 \%(15 / 54)$ there was 2 or more alterations present. The metabolic disorders most frequently observed were hypercalciuria (15/54) 27,7\%,, hypocitraturia (15/54) 27,7\%., hyperuricemia (8/54) 14,8\%. and hyperoxaluria (8/54) $14,8 \%$. There was no significant difference in age or sex between the groups with and without metabolic abnormality.

Conclusions: Most patients with recurrent lithiasic pathology or at high-risk display one or more metabolic disorders, being hypercalciuria and hypocitraturia the most frecuently encountered. In this study, there was no difference between sexes in most of the metabolic disorders, nor in its age distribution. These results demonstrate the need for metabolic studies in high-risk patients, since there are tools that allow therapeutic medical management of metabolic disorders and thus reduce the recurrence of lithiasis.
\end{abstract}

Keywords: Lithiasis. Metabolic disease. Metabolic study. 
T a patología urolitiásica es altamente prevalente $y$ varía de un $1 \%$ a un $15 \%$ dependiendo de múltiples factores, siendo el doble más frecuente en hombres que en mujeres ${ }^{1,2}$. Se ha demostrado en algunas series que el riesgo de recurrencia luego de un primer episodio es de hasta un $70 \%$ en períodos de seguimiento de hasta 20 años, y de los cuales la mitad recurriría antes de los 5 años $^{3}$. La tendencia a la recurrencia de esta enfermedad hace necesario que la enfrentemos no sólo en el episodio agudo que implica la remoción del cálculo, sino que también en su prevención para lo cual es indispensable un estudio metabólico apropiado. La prevalencia de los distintos trastornos metabólicos en la génesis de la litiasis varía de un país a otro, de acuerdo a variables tanto dietéticas como raciales y geográficas ${ }^{4-6}$.

El objetivo de este trabajo es evaluar la prevalencia de las alteraciones metabólicas en un grupo selecto de pacientes con patología urolitiásica considerados de alto riesgo, ya sea por criterios de recurrencia, magnitud de la patología al momento del diagnóstico o las serias consecuencias que tendría un nuevo episodio (paciente monorreno).

Un segundo objetivo de este trabajo es analizar las diferentes alteraciones metabólicas y su impacto según edad y género.

\section{MATERIAL Y MÉTODOS}

Se realizó un estudio descriptivo de 54 pacientes, 37 hombres y 17 mujeres, portadores de patología urolitiásica de alto riesgo. Se usó como criterios de inclusión aquellos pacientes con litiasis recurrente en un período inferior a 5 años (26 pacientes), nefrolitiasis múltiple uni o bilateral o coraliforme (14 pacientes), con un primer episodio antes de los 25 años de edad (10 pacientes), con riñón único (3 pacientes) o radiolúcida en un paciente (Tabla 1).

El estudio metabólico que se realizó a todos los pacientes consistió en: calcemia, uricemia, fosfemia, PTH sérica, calciuria/24 h, uricosuria/24 h, oxalaturia/24 h, citraturia/24 h y fosfaturia/24 h. Los valores obtenidos fueron ajustados de acuerdo a la creatininuria y peso, considerándose una recolección urinaria óptima con una creatininuria calculada de $20 \mathrm{mg} / \mathrm{kg}$ peso $/ 24 \mathrm{~h}$. Los pacientes con hipercalciuria se clasificaron de acuerdo a los valores séricos de PTH. Los pacientes con PTH sérica normal o baja se clasificaron como hipercalciuria absortiva. Los pacientes con PTH sérica elevada recibieron recibieron hidroclorotiazida $50 \mathrm{mg} /$ día
Tabla 1

\begin{tabular}{lcc}
\hline Variables & N & \% \\
\hline Sexo & & \\
$\quad$ Hombres & 37 & 68,5 \\
$\quad$ Mujeres & 17 & 31,4 \\
Indicación de estudio & & \\
$\quad$ Recurrente & 26 & 48,15 \\
$\quad$ Bilateral & 12 & 22,22 \\
Coraliforme & 2 & 3,7 \\
Monorreno & 3 & 5,56 \\
$\quad$ Radiolucida & 1 & 1,85 \\
$\quad$ Menor de 25 años & 11 & 18,52 \\
& & \\
Tratamiento & & \\
LEC & 36 & 66,67 \\
$\quad$ Espontánea & 8 & 14,82 \\
$\quad$ Nefrolitectomía percutánea & 4 & 7,41 \\
$\quad$ Ureteroscopía & 3 & 5,56 \\
Catéter Pigtail & 1 & 1,85 \\
$\quad$ Nefrectomía & 1 & 1,85 \\
$\quad$ Litectomia abierta & 1 & 1,85 \\
\hline
\end{tabular}

durante 2 semanas y se midieron nuevamente los valores séricos de PTH. Aquellos pacientes cuya PTH sérica se normalizó se clasificaron como hipercalciuria renal y aquellos cuya PTH sérica persistió elevada se clasificaron como hiperparatiroidismo primario.

Para el análisis estadístico se utilizó el programa estadístico STATA 7.0. Las variable se valuaron con t-student y se estimó la significancia estadística con un $\mathrm{p}<0,05$.

\section{RESULTADOS}

En el 64,8\% (35/54) de los pacientes con urolitiasis de alto riesgo se observó alguna alteración metabólica. En este grupo, el 37\% (20/54) presentó sólo una alteración metabólica. El 27,7 \% (15/54) presentó 2 ó más alteraciones. Sólo un paciente $(1,8 \%)$ presentó una combinación de 4 alteraciones metabólicas (Fig. 1).

Las alteraciones metabólicas en orden de frecuencia fueron:

- Hipercalciuria (15/54) 27,7\%.

- Hipocitraturia (15/54) 27,7\%.

- Hiperuricemia (8/54) 14,8\%.

- Hiperoxalaturia (8/54) $14,8 \%$.

- Hiperuricosuria (7/54) $12,9 \%$.

De los pacientes con hipercalciuria destacan 7 $(12,9 \%)$ de origen absortivo, $7(12,9 \%)$ de origen renal y uno $(3 \%)$ con hiperparatiroidismo primario (PTH sérica muy elevada sin variación post hidro- 


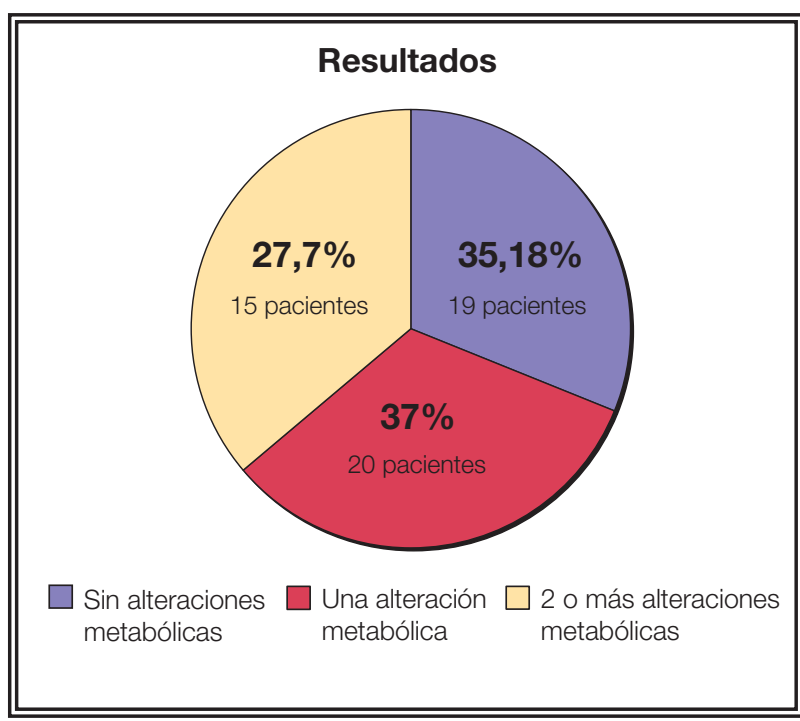

\section{FIGURA 1}

Tabla 2

\section{Combinación de trastornos} metabólicos

$\mathbf{N}^{\circ}$ pacientes

\begin{tabular}{ll}
\hline Hiperuricemia + Hipocitraturia & 3 \\
Hiperuricemia + Hiperuricosuria & 2 \\
Hipercalciuria + Hiperoxalaturia & 3 \\
Hiperoxalaturia + Hipocitraturia & 2 \\
Hipercalciuria + Hiperuricemia & 2 \\
Hipercalciuria + hiperuricosuria & 1 \\
Hipercalciuria + Hipercalcemia & 1 \\
Hipercalciuria + Hiperuricemia + & \\
Hiperuricosuria + Hiperoxalaturia & 1 \\
\hline
\end{tabular}

clorotiazida). Sólo hubo dos pacientes con hipercalcemia, uno correspondió al paciente con hiperparatiroidismo primario. La fosfemia y la fosfaturia fue normal en la totalidad de los pacientes. En el grupo que presentó trastornos metabólicos asociados, se observaron prácticamente todas las combinaciones posibles (Tabla 2).

$\mathrm{El}$ rango de edad de este grupo de pacientes fue de 15 a 75 años, con un promedio de 42,9 (SD: $\pm 13,85$ ) años (Tabla 3). Al evaluar el impacto de la edad no se observa una diferencia estadísticamente significativa entre los grupos con y sin trastornos metabólicos (Fig. 2). En relación al sexo, no hubo diferencia estadísticamente significativa en la distribución de las distintas alteraciones metabólicas entre hombres y mujeres. En este estudio, sólo la hiperoxalaturia mostró una tendencia con predominio en el grupo de hombres (Fig. 3).

\section{DISCUSIÓN}

La mayoría de los pacientes con patología litiásica recurrente o de alto riesgo presentan una o más alteraciones metabólicas, predominando la hipercalciuria y la hipocitraturia.

En nuestro estudio no hubo diferencia entre ambos sexos en la mayoría de las alteraciones metabólicas, ni tampoco en la distribución etaria. Si embargo, es importante destacar que la casuística es limitada con sólo 54 pacientes, y por lo tanto, la ausencia de significancia estadística debe interpretarse en este contexto.

Tabla 3

\begin{tabular}{|c|c|c|c|c|c|}
\hline Variable & $\mathbf{N}$ & $\mathbf{X}+/-\mathbf{S D}$ & Min & $\operatorname{Max}$ & $\mathbf{P}$ \\
\hline \multicolumn{6}{|l|}{ Sexo } \\
\hline Hombre & 37 & 68,5 & & & \\
\hline Mujeres & 17 & 31,4 & & & \\
\hline Edad & & $42,9(13,85)$ & 15 & 75 & 0,376 \\
\hline Hombres & & $43,35(13,44)$ & 20 & 75 & \\
\hline Mujeres & & $42,05(15,09)$ & 15 & 72 & \\
\hline \multicolumn{6}{|c|}{ Alteración Metabólica (Resultados y análisis por sexo) } \\
\hline Hipercalcemia & 2 & $9,37(0,48)$ & 8,5 & 11 & 0,566 \\
\hline Hiperuricemia & 8 & $5,44(1,52)$ & 2,4 & 9,2 & 0,669 \\
\hline Hiperfosfemia & 0 & $3,42(0,5)$ & 2,3 & 4,3 & 1,0 \\
\hline Hipercalciuria & 15 & $203,59(106,83)$ & 10 & 468 & 0,637 \\
\hline Hiperoxalaturia & 8 & $26,16(11,84)$ & 8 & 70 & 0,210 \\
\hline Hiperuricosuria & 7 & $487,53(267,4)$ & 10 & 1700 & 0,487 \\
\hline Hipocitraturia & 15 & $503,07(255,1)$ & 71 & 1680 & 0,403 \\
\hline Hiperfosfaturia & 0 & $715,4(254,75)$ & 107 & 1300 & 1,0 \\
\hline $\mathrm{Ph}$ Urinario & 0 & $5,94(0,23)$ & 5 & 6,5 & 1,0 \\
\hline PTH & 11 & $53,72(40,34)$ & 6 & 283 & 0,736 \\
\hline
\end{tabular}






FIGURA 2

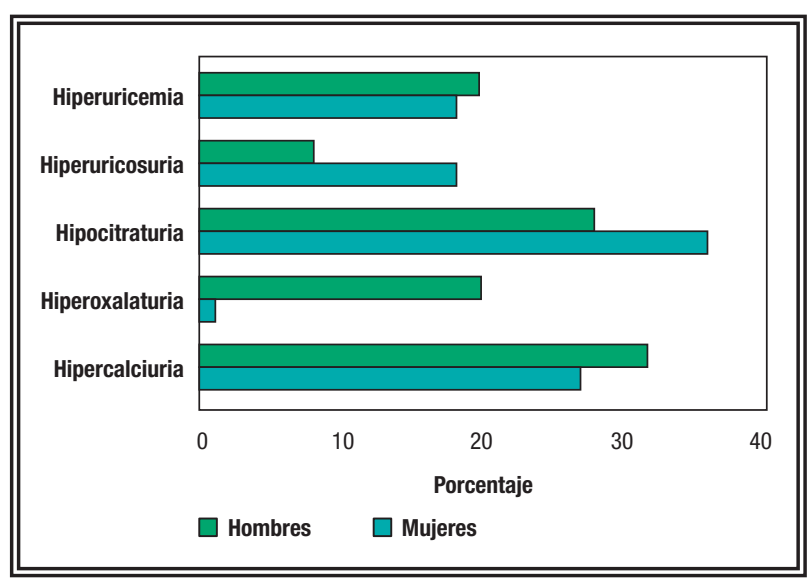

FIGURA 3

Nuestros resultados en relación con experiencias latinoamericanas son similares a los encontrados por Massari ${ }^{7}$ en Argentina (77\% de trastornos metabólicos), pero inferiores a los descritos por Weisinger $^{8}$ en Venezuela $(88 \%$ de trastornos metabólicos).

En el 37\% se presentó un trastorno metabólico único y en el 27,7\% un trastorno combinado, a diferencia de lo hallado por Levy et al. ${ }^{9}$ donde hubo un claro predominio de los trastornos combinados (58\%).

Finalmente, este es un trabajo descriptivo en un grupo de alto riesgo de litiasis, sin un grupo control, y en consecuencia no podemos ni pretendemos medir el real impacto de cada alteración metabólica en la génesis de la patología litiásica.

\section{CONCLUSIÓN}

Estos resultados demuestran la necesidad de realizar estudios metabólicos en pacientes con urolitiasis de alto riesgo, dada su alta prevalencia, y que además, actualmente existen herramientas terapéuticas específicas (dietéticas y medicamentosas) para cada trastorno con aceptables grados de eficacia ${ }^{10,11}$. Este grupo de pacientes está actualmente en seguimiento para evaluar la recurrencia de litiasis bajo terapia específica para cada alteración metabólica encontrada y será dado a conocer con 5 años de seguimiento.

\section{REFERENCIAS}

1. Robertson WG, Peacock M, Baker M, Marshall DH, Pearlman B, Speed R. Studies on the prevalence and epidemiology of urinary stone disease in men in Leeds. $\mathrm{Br} \mathrm{J}$ Urol. 1983 Dec;55(6):595-598

2. Andersen DA. Environmental factors in the aetiology of urolithiasis. In Urinary Calculi 1973; 130-144.

3. Hans-Goran Tiselius. Stone incidence and prevention. Brazilian Journal of Urology (2000), 26: 452-462.

4. Robertson WG, Peacock M, Hodgkinson A. Dietary Changes and the incidence of urinary calculi in the U.K. between 1958 and 1976. J Chronic Dis. 1979;32(6):469-476.

5. Andersen DA. Historical and geographical differences in the pattern of incidence of urinary stones considered in relation to possible aetiological factors. En: Proc. Renal Stone. London, Churchill 1969,7-31.

6. Pyrah LN. Epidemiology of urolithiasis. En: Renal Calculus. New York Springer -Verlag; 1979, 3-17.

7. Massari PU, Garay G, Holz RM. Eperiencia Latino Americana em Litíase renal. Córdoba. En: Calculose Renal. Fisiopatología, Diagnóstico, Tratamiento. Sao Paulo; Sarvier 1995,342-346.

8. Weisinger JR, Vellorí Font E. Experienia Latino Americana em Litíase renal. Venezuela. En: Calculose Renal. Fisiopatología, Diagnóstico, Tratamiento. Sao Paulo; Sarvier 1995,354-359.

9. Levy FL, Adams-Huet B, Pak CY. Ambulatory evaluation of nephrolithiasis : an update of a 1980 protocol. Am J Med. 1995 Jan;98(1):50-59.

10. Bijan Shekarriz, Marshall L. Stoller. Metabolic evaluation of stone disease. Brazilian Journal of Urology 2001; 27,10-18.

11. Pak CY, Peters P, Hurt G, Kadesky M, Fine M, Reisman D et al. Is selective therapy of recurrent nephrolithiasis possible?. Am J Med. 1981; Oct;71(4):615-22.

Correspondencia autor: Dr. Enrique Ossandón Salas Servicio de Urología. Unidad de Litiasis. Hospital Clínico de la Universidad de Chile E-mail autor: enriqueossandon@mi.cl Información artículo: Original - Litiasis Trabajo recibido: septiembre 2008

Trabajo aceptado: enero 2009 